\title{
Texture Description with Completed Local Quantized Patterns
}

\author{
Xiaohua Huang ${ }^{1}$, Guoying Zhao ${ }^{1}$, Xiaopeng Hong ${ }^{1}$, Matti Pietikäinen ${ }^{1}$, \\ and Wenming Zheng ${ }^{2}$ \\ ${ }^{1}$ Center for Machine Vision Research, Department of Computer Science and Engineering, \\ University of Oulu, Finland \\ 2 Research Center for Learning Science, Southeast University, China \\ \{huang.xiaohua, gyzhao, xhong, mkp\}@ee.oulu.fi, \\ wenming_zheng@seu.edu.cn \\ http://www.cse.oulu.fi/cmv
}

\begin{abstract}
Local binary patterns (LBP) has been very successful in a number of areas, including texture analysis and face analysis. Recently, local quantized patterns (LQP) was proposed to use vector quantization to code complicated patterns with a large number of neighbors and several quantization levels. It uses lookup table technique to map patterns into the corresponding indices. In this paper, we propose completed local quantized patterns (CLQP) for improving the performance of LQP. Firstly, we find that LQP only considers the sign-based difference, it thus misses some discriminative information. We therefore propose to use the magnitude-based and orientation-based differences to complement the sign-based difference for LQP. We finally use vector quantization to learn three separate codebooks for local sign, magnitude and orientation patterns, respectively. Secondly, we also observe that LQP uses random initialization in vector quantization, this leads to losing the distribution of local patterns and costing much computational time. For reducing the unnecessary computational time of initialization, we use preselected dominant patterns as the initialization. Our experimental results show that CLQP outperforms well-established features including LBP, LTP, CLBP, LQP on a range of challenging texture classification problems and an infant pain detection problem.
\end{abstract}

Keywords: Local binary pattern, local orientation, magnitude, texture descriptor, pain detection.

\section{Introduction}

Local binary patterns (LBP) [12] is considered as an effective descriptor in a number of areas including texture classification [12], face analysis [1] and medical image analysis [11]. Since the 1990's, many variants of LBP [14] have been proposed. Some representative ones include local ternary patterns (LTP) [15], a completed model of LBP (CLBP) [7], and center-symmetric LBP (CS-LBP) [10].

Recently, an interesting texture descriptor, called local quantized patterns, was proposed to be used in texture classification and object detection [8]. In LQP, two important issues of LBP were considered: (1) how to code some complicated patterns and 
(2) how to find the representative and most discriminative patterns. In practice, some complicated patterns, especially the sampling patterns with a large number of neighbors and several quantization levels, will lead to high dimensional histograms. On the other hand, the typical coding, such as uniform code [12], could be restricted by the exponential growth of the codebook size with neighborhood size and quantization depth [8]. For solving these problems, LQP applies vector quantization to code some complicated patterns and produce the dominant patterns. Furthermore, lookup-table technique is used to replace the nearest neighbor search method for allowing real-time coding. These simple methods make LQP more robust and discriminative.

However, there are two major limitations of LQP. The first one is that LQP only exploits the sign-based difference. However, supplementary information, like magnitude, can be useful. In [7], a completed model of LBP (CLBP) was proposed to incorporate with three complementary components (CLBP): the sign-based difference, the magnitude-based difference, and central pixel intensity. In practice, it is known that the central pixel intensity information is very sensitive to illumination changes [21]. In recent years, some state-of-the-art works show that the orientation is useful and attractive information for a feature descriptor. For example, Zhang et al. [20] proposed Histogram of Gabor Phase Pattern combining the spatial histogram and the Gabor phase information coding scheme. Xie et al. [19] proposed local Gabor XOR patterns, which encodes the Gabor phase by using local XOR pattern. Vu and Caplier [18] presented multiple features combining patterns of oriented edge magnitude and patterns of domination orientations. Inspired by the above-mentioned works, we therefore propose a completed local quantized pattern operator (CLQP) to extend LQP by using the sign-based difference, the magnitude-based difference and the orientation-based difference.

Another limitation of LQP is that the initialization of vector quantization is not stable. Typically, the common way is that some samples are randomly chosen as the initialization. But it needs to be repeated several times for convergence. In our study, we observe that the patterns of LBP and CLBP have one interesting property: some patterns occur many times. This property is used to develop some novel methods, such as dominant LBP [9] and discriminative LBP [6]. This property also motivates us to develop the other way to initialize vector quantization. In our study, patterns that occur many times are regarded as predominating patterns. They can be viewed as the distribution of the texture or appearance.

To address the limitations of LQP mentioned above, we propose that (1) a new descriptor incorporating the sign-based difference, magnitude-based difference and orientation-based difference into steps of LQP, and (2) the revised vector quantization step of LQP by using a dominant-pattern method.

The paper is organized as follows. In Section 2, we briefly review the completed model of local binary pattern, and present local orientation pattern and revised vector quantization method. In Section 3 we carry out experiments dealing with the applications of CLQP in texture classification and infant pain detection. Section 4 provides concluding remarks and possible extensions. 


\section{Completed Local Quantization Pattern}

The essence of the proposed texture descriptor is to characterize texture or appearance by using the sign-based, magnitude-based, and orientation-based differences. The procedure of the proposed algorithm consists of two components: the learning and inference stages. The learning procedure is illustrated in Fig 1 It includes four stages: (1) three kinds of information (local sign, magnitude and orientation patterns) are extracted from the image, in which local orientation pattern is realized by using orientation estimation and quantification, (2) three separate codebooks (called O, S, and M, respectively) are learned by using vector quantization, (3) the sign, magnitude and orientation patterns are mapped into their corresponding codebook by using lookup table (LUT), and (4) three histograms are concatenated into one vector. The inference stage consists of all stages except the second stage. In our case, we consider four neighborhood topologies, as shown in Fig 2 Figs 2 a-c) show three common topologies with circular neighborhoods of $P$ sampling points and radius $R$ around the central pixel. In Fig 2(d), the multi-ring topology $\left(\right.$ Disc $_{5}$ ) has two rings with 24 sampling points in total around the central pixel.

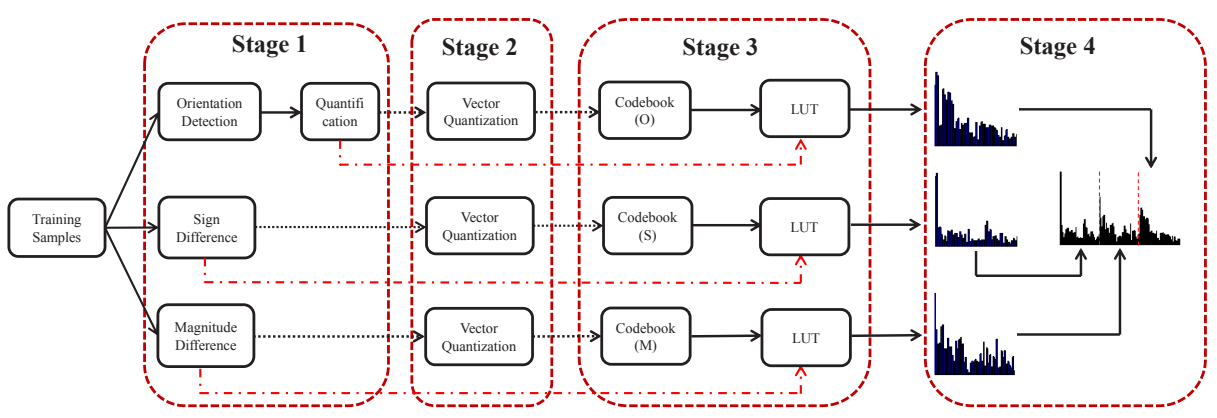

Fig. 1. Overview of the proposed algorithm

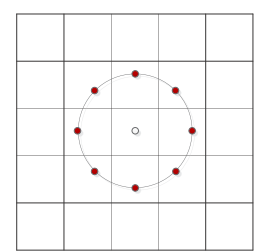

(a)

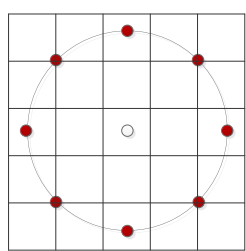

(b)

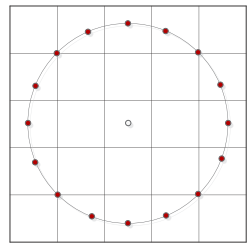

(c)

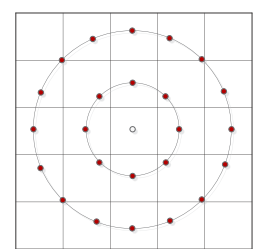

(d)

Fig. 2. Three circular neighborhoods with $P$ sampling points and radius $\mathrm{R}$ around the central point (a-c), and Disc 5 neighborhoods (d). (a) $P=8, R=1$, (b) $P=8, R=2$, (c) $P=16, R=2$, (d) $P=24$. The pixel values are bilinearly interpolated whenever the sampling point is not in the center of a pixel. 


\subsection{Review of CLBP}

CLBP is an important local descriptor incorporating sign-based difference and magnitude-based difference [7]. Here we briefly review CLBP. Consider a monochrome image $I(x, y)$ and let $g_{c}$ denote the gray level of any arbitrary pixel $(x, y)$. Moreover, let $g_{p}(p=0,1, \ldots, P-1)$ denote the gray value of $\mathrm{P}$ sampling points in local neighborhoods around $(x, y)$. The difference between the center pixel and its surrounding pixel can be calculated as $d_{p}=g_{p}-g_{c}$. The difference can be further decomposed into sign $u_{p}$ and magnitude $v_{p}$ as follows,

$$
d_{p}=u_{p} * v_{p}=\operatorname{sign}\left(d_{p}\right) *\left|d_{p}\right|
$$

where $u_{p}=\left\{\begin{array}{l}1, d_{p} \geq 0 \\ 0, d_{p}<0\end{array}\right.$ is the sign of $d_{p}$ and $v_{p}$ is the magnitude of $d_{p}$.

The sign pattern of $(x, y)$ has the same formulation (binary) as the LBP operator. It can be represented as $\left[u_{0}, \ldots, u_{P-1}\right]$. For magnitude pattern $\left[v_{0}, \ldots, v_{P-1}\right]$, it is simply converted into a consistent format with that of sign pattern by a threshold $\delta$. Here we set it as the mean value of $v_{p}$ from the whole image. The magnitude pattern can be converted as $h_{p}=\left\{\begin{array}{l}1, v_{p} \geq \delta \\ 0, v_{p}<\delta\end{array}\right.$. Therefore, the magnitude pattern can be rewritten as $\left[h_{0}, \ldots, h_{P-1}\right]$.

\subsection{Local Dominant Orientation Patterns}

The basic idea of difference of orientation is to encode the relationships between dominant orientations of neighboring pixels in the image. In our algorithm, the procedure of the difference of orientation can be divided into the following stages: (1) the estimation of orientation angle of the pixel, (2) the calculation of dominant orientation, and (3) the operation on neighborhood orientations.

We must firstly estimate the orientation angle of a pixel. To calculate this, the horizontal and vertical filters are generally used to approximate the orientation of an image patch around the pixel [3]. But they are limited to small patches. With the patch size increasing, another way [18] can be developed by using accumulated gradient over all pixels. In this method, the gradient vector of each pixel is calculated, and then a local histogram of gradient over all pixels of one patch is accumulated. Finally the orientation angle is chosen according to the largest histogram bin. Instead, other possible ways, such as Gabor filters and Gaussian recursive transformation, have been proposed to estimate the orientation angle of each pixel [5[19|20]. The advantage is that they could be in practice applied to various patch sizes.

Following the work of [5], a Gaussian kernel is used to estimate the orientation angle of the image patch. For each Gaussian kernel, we make the multi-ring circular mask (similar to Disc ${ }_{5}$ shown in Fig 2). Let the size of an image patch denote as $D \times D$, and the circular mask includes $\left\lfloor\frac{D}{2}\right\rfloor$ circles, where $\lfloor x\rfloor$ means the floor of $x$. Each ring has radius $r$ and $8 \times r$ sampling points around a central point, where $r=1, \ldots,\left\lfloor\frac{D}{2}\right\rfloor$. We denote the number and interval of the orientation bins as $M$ and $\Delta \theta$, respectively, where $\Delta \theta=\frac{2 \pi}{M}$. We know that the angle of each orientation bin can be estimated as 
$\theta_{m}=m \times \Delta \theta, m=0, \ldots, M-1$. For $\theta_{m}$, its Gaussian kernel is defined as the difference between two oriented with shifted kernels in an image patch,

$$
G_{\theta_{m}}=\frac{G_{\theta_{m}}^{-}-G_{\theta_{m}}^{+}}{\sum_{x, y}\left[\left(G_{\theta_{m}}^{-}-G_{\theta_{m}}^{+}\right) \cdot h\left(G_{\theta_{m}}^{-}-G_{\theta_{m}}^{+}\right)\right]},
$$

where

$$
\begin{aligned}
G_{\theta_{m}}^{-}= & \frac{1}{2 \pi \sigma^{2}} \exp \left(-\frac{\left(x-\sigma \cos \theta_{m}\right)^{2}+\left(y-\sigma \sin \theta_{m}\right)^{2}}{2 \sigma^{2}}\right), \\
G_{\theta_{m}}^{+}= & \frac{1}{2 \pi \sigma^{2}} \exp \left(-\frac{\left(x+\sigma \cos \theta_{m}\right)^{2}+\left(y+\sigma \sin \theta_{m}\right)^{2}}{2 \sigma^{2}}\right), \\
& h\left(G_{\theta_{m}}^{-}-G_{\theta_{m}}^{+}\right)=\left\{\begin{array}{l}
1, G_{\theta_{m}}^{-}-G_{\theta_{m}}^{+}>0 \\
0, G_{\theta_{m}}^{-}-G_{\theta_{m}}^{+} \leqslant 0
\end{array}\right.
\end{aligned}
$$

and $\sigma$ is a root mean square deviation of the Gaussian distribution, $\theta_{m}$ is the angle of the Gaussian rotation, $(x, y)$ is the coordinate of circular mask. For this image patch, the response of each orientation can be obtained by convolving with each Gaussian kernel. We can estimate the orientation angle of each pixel according to the maximum response of Gaussian kernel.

As mentioned above, the orientation angle of each pixel has displaced the gray information. Motivated by the formulation of LBP, we try to find out a method for exploiting the relationship of orientation angles of the pixel and its surrounding neighbors. Based on any neighborhood topology (in Fig.2), we assume that the orientation angles of the center pixel $\left(x_{c}, y_{c}\right)$ and its neighbors $\left(x_{p}, y_{p}\right)$ are $\theta\left(x_{c}, y_{c}\right)$ and $\theta\left(x_{p}, y_{p}\right)$, respectively. In general, we could compute the difference of orientation angle [16] and subsequently code it as $0 / 1$ by setting a threshold. However, it is difficult to find the best threshold. Besides that, the threshold will lead the algorithm to be complicated and instable. Instead, for stability, we further quantify the orientation angle. In our case, we apply the quantification function [3] as follows,

$$
t=\bmod \left(\left\lfloor\frac{\theta(x, y)}{\frac{2 \pi}{T}}+0.5\right\rfloor, T\right),
$$

where $T$ is the number of dominant orientation.

We assume that the dominant orientation bin of the pixel $\left(x_{c}, y_{c}\right)$ and its surrounding pixels $\left(x_{p}, y_{p}\right)$ can be denoted as $t_{c}$ and $t_{p}$. Their relationship is calculated as follows,

$$
o_{p}=t_{c} \bigoplus t_{p}=\left\{\begin{array}{l}
0, t_{c}=t_{p} \\
1, t_{c} \neq t_{p}
\end{array},\right.
$$

therefore, the orientation pattern can be written as $\left[o_{0}, \ldots, o_{P-1}\right]$, where $P$ is the number of the sampling points.

In the above-mentioned procedure, we know that $M$ and $T$ are two important parameters in orientation pattern. Let us discuss the property of these two parameters. (1) The orientation angle estimation is sensitive to $M$. If $M$ is too small, it will cause inaccurate orientation angle estimation. Thus, $M$ is as large as possible for estimating 
accurately the orientation angle. However, if $M$ is too large, it will lead to unnecessary computation. (2) In [19], Xie et al. showed that the appropriate quantification level could achieve a good balance between robustness to orientation variation and representation power of local patterns. In [18], Vu and Caplier discussed how the quantification level could affect the local pattern for the orientation in neighbor structure. Thus, $T$ performs a trade-off role between the robustness to orientation variation and representation power of local patterns. Inspired by [5]19], we set $M$ and $T$ as 16 and 4, respectively.

\subsection{Revision of Vector Quantization}

Typically, the coding of local pattern can be realized by a predefined pattern model [12]. A recent work [8] proposed to use vector quantization for codebook learning for local patterns. It can be applied to both simple and complicated neighborhood structures as shown in Fig 2 The advantages of vector quantization include that (1) it is a generalized form of local pattern feature and (2) it uses a learned codebook to make coding fast. We therefore exploit it to produce the codebook for the three above-mentioned local patterns.

Typically, in visual recognition, the local patterns from all images are fed into kmeans clustering, and then the codebook is learned. With the number of local patterns increasing, powerful hardware and huge amount of memory are needed. In our study, we find that the local patterns of sign, magnitude and orientation are binary vectors. Some patterns often occur many times. For example, for neighborhood sampling structure with radius one and eight sampled points, there are 256 possible code values for local pattern of sign, magnitude and orientation. In order to speed up the clustering procedure, we compute the weights of occurred patterns, and then feed them into the clustering. In our experiments, we could find that this trick has good performance and reduces much the computational time.

Through our study, the initialization problem is critical to vector quantization based on k-means clustering. It is generally realized by randomly choosing the samples from the training ones. But it needs to be repeated many times for finding the best initialization. Motivated by the benefit of dominant patterns [9], we propose to use the dominant patterns of the images replacing the random initialization.

Let $C$ be the codebook size of vector quantization, we accumulate the occurrence of local patterns $w_{1}, w_{2}, \ldots, w_{n}$ from all training samples, and then sort the local patterns by the descending order of occurrence. We select the $C$ local patterns as the dominant ones. Instead of random initialization, we feed these dominant patterns to k-means clustering. Fig 3 shows the performance of our dominant-pattern approach and random initialization method (10-time repeated)for the local sign-based pattern in Brodatz32 database [13]. They were carried out on Matlab R2012 64 bits with 2.5GHz Intel Xoen E5-2640 processor and 128Gb of RAM. From this figure, we can see that the classification accuracy by dominant-pattern initialization is better than the former one. We can also observe that the gain in computational time of random initialization method is proportional to the number of random initializations, and dominant-pattern approach needs less time. 


\subsection{Feature Descriptor and Classification}

From the previous section, three kinds of pattern are derived: the sign-based pattern $(S)$, magnitude-based pattern $(M)$, and orientation-based pattern $(O)$. Finally, we use the revised vector quantization to learn the three separate codebooks. The advantage is that we can explore the discriminative and most representative codebook for each one. For each verified image, we can get their histograms through mapping three patterns into the codebook. Here, we simply concatenate them into one histogram. For briefly, we denote it as $\mathrm{CLQP}_{S / M / O}$. In our method, unless specified otherwise, the three-nearestneighbor classifier with Chi-square distance measure is used for fair comparison.

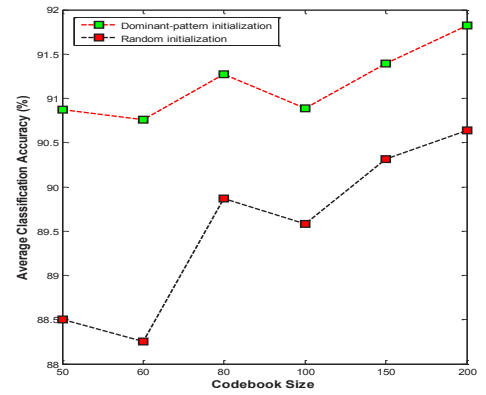

(a)

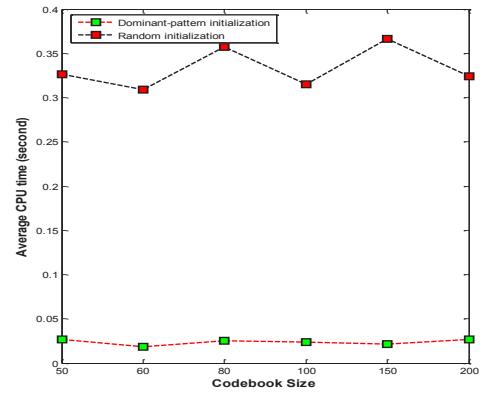

(b)

Fig. 3. The comparative performance of dominant-pattern initialization and random initialization (10 times repeated) on Brodatz 32 dataset [13]. It is repeated by 10 -fold-cross-validation. (a) Average classification accuracy (\%), (b) average CPU time (seconds).

\section{Experiments}

In this section, we use our method for texture classification and emotion recognition, and compare the performance with those of the state-of-the-art methods.

\subsection{Texture Classification Experiments}

Texture classification plays an important role in many applications. Several approaches to the extraction of the texture features have been proposed. Experiments are carried out on three different texture databases: Brodatz 32 [13], KTH-TIPS2-a [2] and CUReT [4]. For Brodatz32, it comprises 2,048 samples, with 64 samples in each of the 32 texture categories. In CUReT, there are 5612 texture images, with 92 samples in each of the 61 real-world surfaces. The KTH-TIPS2-a texture dataset contains 11 texture classes with 4,395 images. In our experiments, we choose gray images for CUReT and transform color images of size $200 \times 200$ pixels into gray level for KTH-TIPS2-a.

We follow the experimental setups of [3]2/4[13]. For Brodatz32, the performance of our method was evaluated with ten different randomly chosen training and test sets [3]13]. The data for each class is randomly split into equal halves with one used for 
training and the other one for testing. For CUReT, we split the images of each class into equal parts and use half for training and the remaining half for testing, and we report averages over 10 random trials [17]. For KTH-TIPS2-a, three samples of each class are used for training and the fourth for testing, and we report averages over four random partitions [2].

The average accuracies of different methods are listed in Tab 1 . Since our implementation of LQP was not able to reproduce the results of [8], with our implementation doing a little worse on three cases, we give the results for our implementations (here we denote it $\mathrm{LQP}_{S}$ ) to allow a comparison with the other features. In Tab1 the number of sampling points for LBP, CLBP and LTP is eight, and the radius for LBP and CLBP is one and for LTP two. And Disc ${ }_{5}$ is used in $\mathrm{LQP}_{S}$. From Tab 1, we can observe that $\mathrm{CLQP}_{S / M}$ and CLQP ${ }_{S / M / O}$ outperform LBP, LTP and $\mathrm{LQP}_{S}$ in all three cases.

(1) For Brodatz32, CLQP ${ }_{S / M}$ and $\mathrm{CLQP}_{S / M / O}$ work better than $\mathrm{LQP}_{S}$. They also outperform $\operatorname{CLBP}_{S / M}$ and $\mathrm{CLBP}_{S / M / C}$.

(2) For CUReT, CLQP $S / M / O$ works little worse than $\mathrm{CLBP}_{S / M / C}$ at the average accuracy of $0.42 \%$. It may be because that intensity information can provide more information than orientation in this database. Without the intensity information, $\mathrm{CLQP}_{S / M}$ and $\mathrm{CLQP}_{S / M / O}$ can significantly outperform $\mathrm{CLBP}_{S / M}$.

(3) For KTH-TIPS2-a, CLQP $S / M / O$ achieves the average accuracy of $67.63 \%$. The reproduced result of LQP [8] can get the rate of $64.2 \%$. Our method has better performance than $\mathrm{LQP}$. CLBP $S / M$ and $\mathrm{CLBP}_{S / M / C}$ have worse results compared with $\mathrm{LQP}_{S}$, in turn perform worse than $\mathrm{CLQP}_{S / M}$ and $\mathrm{CLQP}_{S / M / O}$. Furthermore, we can observe that $\mathrm{CLQP}_{S / M / O}$ performs better than $\mathrm{CLQP}_{S / M}$, especially for the KTHTIPS2-a database.

Table 1. Performance for various features and datasets on texture classification. Rows 1-6 show results for our implementations of LBP, CLBP, LTP and LQP (Disc $\mathrm{s}_{5}$ ), Rows 7 and 8 highlight that the performance is produced by optimal local pattern and codebook size. The number in brackets represents the optimal neighborhood topology.

\begin{tabular}{|c|c|c|c|}
\hline$\overline{\text { Method }}$ & Brodatz32 & CUReT & KTH-TIPS2-a \\
\hline$\overline{\mathrm{LBP}}$ & $91.46(1,8)$ & $87.56(1,8)$ & $60.01(1,8)$ \\
\hline $\mathrm{LBP}^{\text {riu } 2}$ & $69.07(1,8)$ & $77.82(1,8)$ & $51.15(1,8)$ \\
\hline$\overline{\operatorname{CLBP}}_{S / M / C}^{r i u 2}$ & $93.83(1,8)$ & $93.34(1,8)$ & $57.14(1,8)$ \\
\hline $\mathrm{CLBP}_{S / M}^{\text {riu2 }}$ & $88.4(1,8)$ & $84.84(1,8)$ & $51.99(1,8)$ \\
\hline$\overline{\text { LTP }}$ & $94.06(2,8)$ & $89.02(2,8)$ & $62.30(2,8)$ \\
\hline $\mathrm{LQP}_{S}$ & $95.54\left(\right.$ Disc $\left._{5}\right)$ & $89.74\left(\right.$ Disc $\left._{5}\right)$ & $63.42\left(\right.$ Disc $\left._{5}\right)$ \\
\hline $\mathrm{CLQP}_{S / M}$ & $96.29\left(\right.$ Disc $\left._{5}\right)$ & $91.97(2,8)$ & $64.35\left(\mathrm{Disc}_{5}\right)$ \\
\hline $\mathrm{CLQP}_{S,}$ & $96.33\left(\right.$ Disc $\left._{5}\right)$ & $92.92(2,8)$ & $67.63\left(\right.$ Disc $\left._{5}\right)$ \\
\hline
\end{tabular}

\subsection{Neonatal Facial Expression Classification on Infant COPE Database}

Recent research on facial expression analysis has provided a protocol for diagnosing the pain of patient, especially for neonates who are incapable of articulating their pain experiences [11]. To access pain states, we conducted experiments on the infant classification of pain expressions (COPE) database. 
4. Dana, K., Ginneken, B., Nayar, S., Koenderink, J.: Reflectance and texture of real-world surfaces. ACM Transactions on Graphics 18(1), 1-34 (1999)

5. Gizatdinova, Y., Surakka, V.: Feature-based detection of facial landmarks from neutral and expressive facial images. IEEE Transactions on Pattern Analysis and Machine Intelligence 28(1), 135-139 (2006)

6. Guo, Y., Zhao, G., Pietikäinen, M.: Discriminative features for texture description. Pattern Recognition 45(10), 3834-3843 (2012)

7. Guo, Z., Zhang, L., Zhang, D.: A completed modeling of local binary pattern operator for texture classification. IEEE Transactions on Image Processing 19(6), 1657-1663 (2010)

8. Hussain, S.u., Triggs, B.: Visual recognition using local quantized patterns. In: Fitzgibbon, A., Lazebnik, S., Perona, P., Sato, Y., Schmid, C. (eds.) ECCV 2012, Part II. LNCS, vol. 7573, pp. 716-729. Springer, Heidelberg (2012)

9. Liao, S., Law, W., Chung, C.: Dominant local binary patterns for texture classification. IEEE Transactions on Image Processing 18(5), 1107-1118 (2009)

10. Heikkilä, M., Pietikäinen, M., Schmid, C.: Description of interest regions with local binary patterns. Pattern Recognition 42(3), 425-436 (2009)

11. Nanni, L., Lumini, A., Brahnam, S.: Local binary patterns variants as texture descriptors for medical image analysis. Artificial Intelligence in Medicine 49(2), 117-125 (2010)

12. Ojala, T., Pietikäinen, M., Mäenpää, T.: Multiresolution gray-scale and rotation invariant texture classification with local binary patterns. IEEE Transactions on Pattern Analysis and Machine Intelligence 24(7), 971-987 (2002)

13. Ojala, T., Valkealahti, K., Oja, E., Pietikäinen, M.: Texture discrimination with multidimensional distributions of signed gray level differences. Pattern Recognition 34(3), 727-739 (2001)

14. Pietikäinen, M., Hadid, A., Zhao, G., Ahonen, T.: Computer Vision using Local Binary Patterns. Springer, Berlin (2011)

15. Tan, X., Triggs, B.: Enhanced local texture sets for face reocgnition under difficult lighting conditions. IEEE Transactions on Image Processing 19(6), 1635-1650 (2010)

16. Tzimiropoulos, G., Zafeiriou, S., Pantic, M.: Subspace learning from image gradient orientations. IEEE Transactions on Pattern Analysis and Machine Intelligence 34(12), 2454-2466 (2012)

17. Varma, M., Zisserman, A.: Classifying images of materials: Achieving viewpoint and illumination independence. In: Heyden, A., Sparr, G., Nielsen, M., Johansen, P. (eds.) ECCV 2002, Part III. LNCS, vol. 2352, pp. 255-271. Springer, Heidelberg (2002)

18. Vu, N.G., Caplier, A.: Mining patterns of orientations and magnitudes for face recognition. In: International Joint Conference on Biometrics, pp. 1-8. IEEE Press, New York (2011)

19. Xie, S., Shan, S., Chen, X., Chen, J.: Fusing local patterns of Gabor magnitude and phase for face recognition. IEEE Transaction on Image Processing 19(5), 1349-1361 (2010)

20. Zhang, B., Shan, C., Chen, X., Gao, W.: Histogram of gabor phase patterns (HGPP): A novel object representation approach for face recognition. IEEE Transactions on Image Processing 16(1), 57-68 (2007)

21. Zhao, G., Ahonen, T., Matas, J., Pietikäinen, M.: Rotation invariant image and video description with local binary pattern features. IEEE Transactions on Image Processing 21(4), 1465-1467 (2012) 